\title{
Determining the Health Literacy Levels and Attitudes of Individuals in Different Countries Towards Vaccination
}

Mehmet Salih YILDIRIM $^{1}$ (D) , Metin YILDIZ $^{2}$ (D) , Abraham TANIMOWO $^{3}$ (D) , Ram Bahadur KHADKA $^{4}$ (D) , Apurba GOHAIN ${ }^{5}$ (D) , Andrianirina RHINO ${ }^{6}$, Nur Ahmad RUDIN ${ }^{7}$, Debopriya GHATAK ${ }^{8}$, Muhammet Ali AYDIN $^{9}$ (D) , Yakup SARPDAGI ${ }^{10}$ (D) , Muhammad TAYYEB $^{11}$ (D) , Kumaran GENGATHARAN ${ }^{12}$, ${\text { Anis } \text { UDDIN }^{13} \text { (D) } \text {, Sweta MAJUMDER }^{14} \text { (D) , Urushi MAGGOON }}^{15}$ (D) , Guray OKYAR ${ }^{16}$ (D)

${ }^{1}$ Vocational School of Health Services, Agri Ibrahim Cecen University School of Health, Agri, Turkey

${ }^{2}$ Department of Nursing, Agri Ibrahim Cecen University School of Health, Agri, Turkey

${ }^{3}$ Institute of Child Health, College of Medicine, University of Ibadan, Oyo State, Nigeria

${ }^{4}$ Tribhuvan University, Province Bagmati, Nepal

${ }^{5}$ Assam University Silchar, Assam, India

${ }^{6}$ Antananarivo, Madagascar

${ }^{7}$ Departmant of Tropical Biology, Faculty of Biology, Universitas Gadjah Mada, Indonesia

${ }^{8}$ Adamas University, India

${ }^{9}$ Faculty of Health Sciences, Ataturk University, Erzurum, Turkey

${ }^{10}$ Ataturk University, Erzurum, Turkey

${ }^{11}$ College of Medical Technology, Medical Teaching institutions, Bacha Khan Medical College, Mardan, Pakistan

${ }^{12}$ Universiti Pendidikan Sultan Idris, Ipoh, Malaysia

${ }^{13}$ L.L.B (Hon's) at Southern University Bangladesh

${ }^{14}$ Youth Leader \& Social Activist pursuing Master of Social Work, India

${ }^{15}$ Univesidad Autonoma Ciudad Juarez, Mexico

${ }^{16}$ Department of Nursing, Agri Ibrahim Cecen University School of Health, Agri, Turkey

Turk J Int Mẹd 2021

DOI: $\underline{10.46310 / \text { tjim. } 850652}$

Keywords: Health literacy, Nationality, Vaccination.

Received:December 31,2020;Accepted: January 30,2021; Published Online: July 29, 2021

Vocational School of Health Services, Agri Ibrahim Cecen University School of Health, 


\section{A B S T R A C T}

Background This research was conducted to determining the health literacy levels and attitudes of individuals in different countries towards vaccination.

Material and Methods This study is cross-sectional, comprised of eight hundred and and thirty eight participants $(n=838)$ drawn from communities in eight different countries; Turkey (Agri), Nigeria (Ibadan), Pakistan (Charsadda), India (Assam), Malaysia (Ipoh), Madagascar (Antananarivo), Indonesia (Bandung), and Nepal (Kathmandu) between September and October 2020.

Results The Health Literacy scale total score mean was 54.92 \pm 23.64 . Vaccine Reliability Scale Total Score Means 55.38 \pm 12.99 . It was found that there was a statistically significant negative correlation between Health Literacy scale total score mean and Vaccine Reliability Scale Total Score Means and two Vaccine Reliability Scale sub dimension; Vaccine Benefit Perception and Trust in Healthcare Professionals.

Conclusions The Health Literacy total score mean was found to be statistically significantly higher among Nepal nationality, male, civil servant and those who do not think the COVID-19 vaccine will be effective and those who do not think they will receive the COVID vaccine if it is free $(p<0.05)$. The vaccine reliability scale total score mean was found to be statistically significantly higher among India nationality, those who receive COVID-19 vaccine, who were not against the COVID-19 vaccine, who think the COVID-19 vaccine will be effective and who will receive the COVID-19 vaccine if it is free $(p<0.05)$. It is recommended to conduct the study in a larger groups.

\section{Introduction}

The availability of vaccines is regarded as one of the cornerstones of the modern world and one of the most important advances in medicine. Although it is known that smallpox patients were vaccinated for the first time in the 1500 s by grinding the scabs of smallpox patients and snorting them; The modern vaccination was carried out by the English doctor Edward Jenner in 1796, when a cattle injected the pus obtained through a smallpox lesion into humans. ${ }^{1}$ Pasteur and Koch also contributed greatly to microbiology in the $19^{\text {th }}$ century and vaccination studies gained even more importance. ${ }^{2}$ The World Health Organization initiated the Global Vaccine Action Plan, which was supported by 194 member countries in 2011 for the spread of vaccination services, and by 2020 , it aimed to deliver the existing vaccines to all communities equally and to prevent morbidities and deaths that may occur in case of inadequate vaccination. ${ }^{3,4}$

Today, when it comes to vaccination and immunization, childhood vaccines still come to mind, but it is known that adults can be protected from deaths, disabilities and various diseases thanks to vaccination and immunization. ${ }^{5}$ Although childhood vaccines are closely monitored by family physicians and offered to the public for free, adult immunization rates are not followed, not offered free of charge to the public, physicians do not update their knowledge on adult vaccination, and the public's knowledge about adult immunization is inadequate. It is stated that the rates are insufficient. ${ }^{6}$ Despite all the known benefits of vaccines, people may exhibit negative attitudes and behavior towards vaccination for reasons such as doubting the safety and efficacy of vaccines, and distrust of governments and healthcare workers. ${ }^{7}$ For this reason, anti-vaccination movements have gained importance in the last 20 years in developed and developing countries, and especially with the spread of the internet, there has been a serious decrease in the number of parents who vaccinate their children.

Insufficient knowledge of parents, who are the main target of anti-vaccine movements, leaves this population vulnerable and open to manipulation. ${ }^{8,9}$ Low level of health literacy (HL), advanced age, being not vaccinated before, and low education level are among the main factors that cause low vaccination knowledge. ${ }^{10-12}$ Health literacy, which is the main modifiable factor that will increase the knowledge level of individuals about immunization and provide a positive change in their attitudes and behavior, draws attention here. 
It is also thought that individuals with high health literacy levels will be able to get information about vaccination and vaccination from the right sources, and as a result, they can take an important place in the fight against vaccine hesitation and vaccine anti-vaccination. ${ }^{13}$ It is known that health literacy, which means gaining the level of knowledge, personal skills and confidence in taking action to improve individual and community health by changing personal lifestyles and living conditions, is directly related to the general health levels of individuals. ${ }^{14}$ In this context, it is obvious that health literacy will play an important role in the maintenance and development of immunization services.

There has been no study in the literature on the health literacy level and attitude towards vaccination of different countries, and it is thought that this study will contribute to literature.

\section{Material and Methods}

\section{Study Design}

This research used a cross- sectional design to determining the health literacy levels and attitudes of individuals in different countries towards vaccination. The countries included in the study were selected with the convenience sampling method.

Area

This study was conducted in selected comunities of eight different countries, the countries and their communities are: Turkey (Agri), Nigeria (Ibadan), Pakistan (Charsadda), India (Assam), Malaysia (Ipoh), Madagascar (Antananarivo), Indonesia (Bandung) and Nepal (Kathmandu).

\section{Population}

The universe of the research comprises of individuals living in Turkey (Agri), Nigeria (Ibadan), Pakistan (Charsadda), India (Assam), Malaysia (Ipoh), Madagascar (Antananarivo), Indonesia (Bandung), and Nepal (Kathmandu). Who are of the age of 18 years and above and English speakers. In the power analysis, the required sample size was calculated as 384 people at $95 \%$ confidence level and 5\% confidence interval. The sample size comprised of eight hundred and thirty eight $(\mathrm{n}=838)$ individuals from the indicated community located in the different countries who agreed to participate in the study.

\section{Data Collection Tools}

An online google form designed by the researcher was used to gather the primary data. The survey comprises of three forms:

1. Demographic characteristics form: It consists of questions on information that can be used to classify the respondents.

2. Vaccine Reliability Scale

It is an 8-item scale developed by Melissa B. Gilkey. ${ }^{15}$ The items are scored between 0 (strongly disagree) and 10 (strongly agree). The scale consists of 3 factors including the benefits of vaccination, the harms of the vaccine, and trust in healthcare providers. The first 4 items are aimed at determining the benefits of the vaccine, Articles 5 and 6 the harms of the vaccine, and Articles 7 and 8 trust in the healthcare worker. A minimum of 0 and a maximum of 80 points can be obtained on the scale. Increasing the total scale score indicates increased confidence in the vaccine. A minimum of 0 and a maximum of 40 points can be obtained from the benefit perception subscale. The higher the score, the higher the perception of benefit. A minimum of 0 and a maximum of 20 points can be obtained from the harm perception subscale. The higher the score, the lower the perception of harm. A minimum of 0 and a maximum of 20 points can be obtained from the trust perception subscale. The higher the score, the higher the trust in healthcare professionals. In our study The Cronbach Alpha value of the scale was found to be 0.78 .

\section{Health Literacy Scale}

Health Literacy Scale, developed by Sorensen (2013). ${ }^{16}$ The Cronbach alpha value of the scale is 0.95 and the Cronbach alpha values of the subscales vary between 0.90 and 0.94 . The scale consists of 25 items and four sub-dimensions. These four subdimensions; Access to Information, Understanding Information, Appraisal/Evaluation, Application/ Use. Access to information sub-dimension includes five items (1-5), the minimum score to be obtained from this subscale is 5 and the maximum score is 25 . Understanding information sub-dimension contains seven items (6-12), the minimum score to be obtained from this subscale is 7 and the maximum score is 35 . The appraisal/evaluation sub-dimension contains eight items (13-20), the minimum score to be obtained from this subdimension is 8 and the maximum score is 40 . The 
application / use sub-dimension contains five items (21-25), the minimum score to be obtained from this subscale is 5 , the maximum score is 25 . The lowest score that can be obtained from the scale is 25 , the highest score is 125 . The higher the score, the higher the health literacy level. The scale, has a five-point likert structure. All items of the scale are positive and there are no negative items. In our study the Cronbach Alpha value of the scale was found to be 0.93 .

\section{Data Collection Methods}

The researcher explained the purpose of the research to the respondents and obtained a verbal consent from the respondents. The online google form prepared by the researchers was then administered to those individuals who agreed to participate in the research.

\section{Limitations}

It is a limitation of this study to be conducted only with those who speak English. The vaccine has some different origins. Like the Russian, German, Chinese vaccine. This may affect vaccine diversity and safety. This is a limitation of the study.

\section{Analysis of Data}

The data analysis was done using the statistical package for social science (SPSS) software. The Primary data was subjected to descriptive statistics of frequency count, percentage, mean and standard deviation. Kolmogorov - Smirnov test was used to test the distribution normality. Mann-Whitney U test was used for binary groups, and the KruskalWallis test was used for groups more than two. Spearman correlation test was used to determine the linear relationship between variables and severity of the relationship. The $p$-value $<0.05$, was accepted as a statistically significant difference.

\section{Ethical Principles}

The study was approved by the University Scientific Research Ethics Committee. The study was conducted in accordance with the ethical standards established in the Declaration of Helsinki.

\section{Results}

Table 1 showed that the study comprises mostly of citizens of Nigeria, Pakistan and India (12.8\% each), $57.5 \%$ were male, $67.3 \%$, were single and $59.5 \%$ of the respondent completed a tertiary education. $42.1 \%$ of the respondent were student. $56.4 \%$ received COVID-19 vaccine, $87.9 \%$ those who do not against the COVID-19 vaccine, $83.7 \%$ those who think the COVID-19 vaccine will be effective and $65.6 \%$ those who will receive the COVID vaccine if it is free. The table also further revealed that the mean age of the respondent was $29.89 \pm 11.41$.

Table 2 revealed that the Health Literacy scale total score mean was 54.92 \pm 23.64 . Access to Information Sub-Dimension 10.76 \pm 5.14 , Understanding Information Sub-Dimension 15.31 \pm 7.03 , Value Setting Sub-Dimension 17.98 \pm 7.81 , Application Sub-Dimension 10.85 \pm 5.03 , Vaccine Reliability Scale Total Score Means 55.38 \pm 12.99 , Vaccine Benefit Perception Sub-Dimension 29.86 \pm 7.78 , Vaccine Harm Perception Sub-Dimension 10.74 \pm 4.87 , Trust in Healthcare Professionals Sub-Dimension $14.77 \pm 4.38$.

Table 3 presented the comparison of respondents' demographic characteristics and their Health Literacy total score mean. The Health Literacy total score mean was found to be statistically significantly higher among Nepal nationality, male, civil servant and those who do not think the COVID-19 vaccine will be effective and those who do not think they will receive the COVID vaccine if it is free $(\mathrm{p}<0.05)$.

Table 4 presented the comparison of respondents' demographic characteristics and their vaccine reliability scale total score mean. The vaccine reliability scale total score mean was found to be statistically significantly higher among India nationality, those who receive COVID-19 vaccine, who do not the COVID-19 vaccine, who think the COVID-19 vaccine will be effective and who will receive the COVID-19 vaccine if it is free $(p<0.05)$.

It was found that there was a statistically significant positive correlation between Health Literacy scale total score mean and all Health Literacy scale sub dimensions and Vaccine Reliability Scale of sub dimension Vaccine Harm Perception $(\mathrm{p}<0.05)$.

It was found that there was a statistically significant negative correlation between Health Literacy scale total score mean and Vaccine Reliability Scale Total Score Means and two Vaccine Reliability Scale sub dimension; Vaccine 
Table 1. Showing the Demographic Characteristics of the Respondents' $(n=838)$

\begin{tabular}{|c|c|c|c|}
\hline Variables & & $\mathbf{n}$ & $\%$ \\
\hline Nationality & $\begin{array}{l}\text { Turkey } \\
\text { Nigeria } \\
\text { Pakistan } \\
\text { India } \\
\text { Malaysia } \\
\text { Nepal } \\
\text { Indonesia } \\
\text { Madagascar }\end{array}$ & $\begin{array}{l}103 \\
107 \\
107 \\
107 \\
97 \\
95 \\
89 \\
133\end{array}$ & $\begin{array}{l}12.3 \\
12.8 \\
12.8 \\
12.8 \\
11.6 \\
11.3 \\
10.6 \\
15.9\end{array}$ \\
\hline Gender & $\begin{array}{l}\text { Female } \\
\text { Male }\end{array}$ & $\begin{array}{l}356 \\
482\end{array}$ & $\begin{array}{l}42.5 \\
57.5\end{array}$ \\
\hline Marital status & $\begin{array}{l}\text { Single } \\
\text { Married }\end{array}$ & $\begin{array}{l}564 \\
274\end{array}$ & $\begin{array}{l}67.3 \\
32.7\end{array}$ \\
\hline Education Level & $\begin{array}{l}\text { Primary education } \\
\text { Secondary education } \\
\text { Tertiary education } \\
\text { Master } \\
\text { Doctorate }\end{array}$ & $\begin{array}{l}34 \\
87 \\
499 \\
205 \\
13\end{array}$ & $\begin{array}{l}4.1 \\
10.4 \\
59.5 \\
24.5 \\
1.6\end{array}$ \\
\hline Occupation & $\begin{array}{l}\text { Civil Servant } \\
\text { Health worker } \\
\text { Pensioners } \\
\text { Business owner/trader } \\
\text { Unemployed } \\
\text { Student } \\
\text { Others }\end{array}$ & $\begin{array}{l}119 \\
50 \\
28 \\
82 \\
104 \\
353 \\
102\end{array}$ & $\begin{array}{l}14.2 \\
6.0 \\
3.3 \\
9.8 \\
12.4 \\
42.1 \\
12.2\end{array}$ \\
\hline Can you receive COVID-19 vaccine? & $\begin{array}{l}\text { Yes } \\
\text { No }\end{array}$ & $\begin{array}{l}473 \\
365\end{array}$ & $\begin{array}{l}56.4 \\
43.6\end{array}$ \\
\hline Are you against the COVID-19 vaccine? & $\begin{array}{l}\text { Yes } \\
\text { No }\end{array}$ & $\begin{array}{l}101 \\
737\end{array}$ & $\begin{array}{l}12.1 \\
87.9\end{array}$ \\
\hline $\begin{array}{l}\text { Do you think the COVID-19 vaccine will } \\
\text { be effective? }\end{array}$ & $\begin{array}{l}\text { Yes } \\
\text { No }\end{array}$ & $\begin{array}{l}701 \\
137\end{array}$ & $\begin{array}{l}83.7 \\
16.3\end{array}$ \\
\hline $\begin{array}{l}\text { Can you receive the COVID vaccine if it } \\
\text { is free? }\end{array}$ & $\begin{array}{l}\text { Yes } \\
\text { No }\end{array}$ & $\begin{array}{l}550 \\
288\end{array}$ & $\begin{array}{l}65.6 \\
34.4\end{array}$ \\
\hline Age (Year) & & \multicolumn{2}{|c|}{$\begin{array}{l}29.89 \pm 11.41 \\
(\min .18, \max .80)\end{array}$} \\
\hline
\end{tabular}

Source: Online field survey 2020.

Benefit Perception and Trust in Healthcare Discussion

Professionals.

It was found that there was a statistically significant negative correlation between Vaccine Reliability Scale total score mean and Health Literacy scale total score mean and all sub dimensions $(p<0.05)$.

It was found that there was a statistically significant positive correlation between Vaccine Reliability Scale total score mean and Vaccine Reliability Scale all sub dimensions $(p<0.05)$.

In this study, the relationship between health literacy and attitude towards vaccination and the findings regarding the situation in different countries are discussed in the light of the literature.

The total mean scores from the Health Literacy Scale were found to be significantly higher among Nepal's people $(p<0.05)$. Health literacy is affected by many factors, which suggests that the awareness level of the individuals in which the study was 
Table 2. Health Literacy and Vaccine Reliability Scale Total Score Means and Sub-

Dimension

\begin{tabular}{lll}
\hline & $\bar{X} \pm$ SD & Min-Max \\
\hline Health Literacy Scale Total Score Means & $\mathbf{5 4 . 9 2 \pm 2 3 . 6 4}$ & $\mathbf{2 5 . 0 0 - 1 2 5 . 0 0}$ \\
Access to Information Sub-Dimension & $10.76 \pm 5.14$ & $5.00-25.00$ \\
Understanding Information Sub-Dimension & $15.31 \pm 7.03$ & $7.00-35.00$ \\
Value Setting Sub-Dimension & $17.98 \pm 7.81$ & $8.00-40.00$ \\
Application Sub-Dimension & $10.85 \pm 5.03$ & $5.00-25.00$ \\
Vaccine Reliability Scale Total Score Means & $\mathbf{5 5 . 3 8} \pm \mathbf{1 2 . 9 9}$ & $\mathbf{4 . 0 0 - 8 0 . 0 0}$ \\
Vaccine Benefit Perception Sub-Dimension & $29.86 \pm 7.78$ & $0.00-40.00$ \\
Vaccine Harm Perception Sub-Dimension & $10.74 \pm 4.87$ & $0.00-20.00$ \\
Trust in Healthcare Professionals Sub-Dimension & $14.77 \pm 4.38$ & $0.00-20.00$ \\
& & \\
\hline
\end{tabular}

Source: Online field survey 2020.

Table 3. Comparison of Individuals' Demographic Characteristics and Health Literacy Total Score Mean

\begin{tabular}{|c|c|c|c|c|}
\hline Variables & & $\mathbf{n}$ & $\bar{X} \pm \mathbf{S D}$ & Statistic \\
\hline Nationality & $\begin{array}{l}\text { Turkey } \\
\text { Nigeria } \\
\text { Pakistan } \\
\text { India } \\
\text { Malaysia } \\
\text { Nepal } \\
\text { Indonesia } \\
\text { Madagascar }\end{array}$ & $\begin{array}{l}103 \\
107 \\
107 \\
107 \\
97 \\
95 \\
89 \\
133\end{array}$ & $\begin{array}{l}51.97 \pm 23.12 \\
45.76 \pm 20.22 \\
51.95 \pm 22.94 \\
52.66 \pm 24.01 \\
55.55 \pm 31.74 \\
75.68 \pm 8.21 \\
58.66 \pm 24.36 \\
50.97 \pm 17.87\end{array}$ & $\begin{array}{l}\mathrm{KW}=128.446 \\
\mathrm{p}=\mathbf{0 . 0 0 0}\end{array}$ \\
\hline Gender & $\begin{array}{l}\text { Female } \\
\text { Male }\end{array}$ & $\begin{array}{l}356 \\
482\end{array}$ & $\begin{array}{l}51.20 \pm 23.14 \\
57.66 \pm 23.66\end{array}$ & $\begin{array}{l}\mathrm{U}=70635.50 \\
\mathrm{p}=\mathbf{0 . 0 0 0}\end{array}$ \\
\hline Marital status & $\begin{array}{l}\text { Single } \\
\text { Married }\end{array}$ & $\begin{array}{l}564 \\
274\end{array}$ & $\begin{array}{l}55.10 \pm 23.04 \\
54.54 \pm 24.87\end{array}$ & $\begin{array}{l}\mathrm{U}=74348.50 \\
\mathrm{p}=0.374\end{array}$ \\
\hline Education Level & $\begin{array}{l}\text { Primary education } \\
\text { Secondary education } \\
\text { Tertiary education } \\
\text { Master } \\
\text { Doctorate }\end{array}$ & $\begin{array}{l}34 \\
87 \\
499 \\
205 \\
13\end{array}$ & $\begin{array}{l}48.94 \pm 27.70 \\
54.49 \pm 23.13 \\
55.85 \pm 23.50 \\
53.46 \pm 23.45 \\
60.38 \pm 23.97\end{array}$ & $\begin{array}{l}\mathrm{KW}=8.145 \\
\mathrm{p}=0.086\end{array}$ \\
\hline Occupation & $\begin{array}{l}\text { Civil Servant } \\
\text { Health worker } \\
\text { Pensioners } \\
\text { Business owner/trader } \\
\text { Unemployed } \\
\text { Student } \\
\text { Others }\end{array}$ & $\begin{array}{l}119 \\
50 \\
28 \\
82 \\
104 \\
353 \\
102\end{array}$ & $\begin{array}{l}62.59 \pm 24.68 \\
59.72 \pm 21.36 \\
47.35 \pm 18.48 \\
52.96 \pm 20.15 \\
49.55 \pm 26.46 \\
55.01 \pm 23.02 \\
52.40 \pm 24.18\end{array}$ & $\begin{array}{l}K W=31.877 \\
p=0.000\end{array}$ \\
\hline Can you receive COVID-19 vaccine? & $\begin{array}{l}\text { Yes } \\
\text { No }\end{array}$ & $\begin{array}{l}473 \\
365\end{array}$ & $\begin{array}{l}53.80 \pm 22.98 \\
56.36 \pm 24.43\end{array}$ & $\begin{array}{l}\mathrm{U}=81449.00 \\
\mathrm{p}=0.161\end{array}$ \\
\hline $\begin{array}{l}\text { Are you against the COVID-19 } \\
\text { vaccine? }\end{array}$ & $\begin{array}{l}\text { Yes } \\
\text { No }\end{array}$ & $\begin{array}{l}101 \\
737\end{array}$ & $\begin{array}{l}58.31 \pm 24.77 \\
54.45 \pm 23.46\end{array}$ & $\begin{array}{l}\mathrm{U}=33783.50 \\
\mathrm{p}=0.132\end{array}$ \\
\hline $\begin{array}{l}\text { Do you think the COVID-19 vaccine } \\
\text { will be effective? }\end{array}$ & $\begin{array}{l}\text { Yes } \\
\text { No }\end{array}$ & $\begin{array}{l}701 \\
137\end{array}$ & $\begin{array}{l}54.00 \pm 23.28 \\
59.62 \pm 24.95\end{array}$ & $\begin{array}{l}\mathrm{U}=41544.50 \\
\mathrm{p}=\mathbf{0 . 0 1 2}\end{array}$ \\
\hline $\begin{array}{l}\text { Can you receive the COVID-19 vaccine } \\
\text { if it is free? }\end{array}$ & $\begin{array}{l}\text { Yes } \\
\text { No }\end{array}$ & $\begin{array}{l}550 \\
288\end{array}$ & $\begin{array}{l}53.59 \pm 24.09 \\
57.44 \pm 22.58\end{array}$ & $\begin{array}{l}U=69469.50 \\
p=0.003\end{array}$ \\
\hline
\end{tabular}

Source: Online field survey 2020. 
Table 4. Comparison of Individuals' Demographic Characteristics and Vaccine Reliability Scale Total Score Mean

\begin{tabular}{|c|c|c|c|c|}
\hline Variables & & $\mathbf{n}$ & $\bar{X} \mathbf{\pm S D}$ & Statistic \\
\hline Nationality & $\begin{array}{l}\text { Turkey } \\
\text { Nigeria } \\
\text { Pakistan } \\
\text { India } \\
\text { Malaysia } \\
\text { Nepal } \\
\text { Indonesia } \\
\text { Madagascar }\end{array}$ & $\begin{array}{l}103 \\
107 \\
107 \\
107 \\
97 \\
95 \\
89 \\
133\end{array}$ & $\begin{array}{l}49.72 \pm 12.97 \\
57.35 \pm 11.12 \\
52.16 \pm 20.15 \\
61.72 \pm 10.87 \\
59.82 \pm 10.90 \\
54.16 \pm 8.71 \\
57.29 \pm 11.13 \\
52.04 \pm 9.93\end{array}$ & $\begin{array}{l}\mathrm{KW}=90.998 \\
\mathrm{p}=0.000\end{array}$ \\
\hline Gender & $\begin{array}{l}\text { Female } \\
\text { Male }\end{array}$ & $\begin{array}{l}356 \\
482\end{array}$ & $\begin{array}{l}56.04 \pm 13.17 \\
54.90 \pm 12.85\end{array}$ & $\begin{array}{l}\mathrm{U}=81324.00 \\
\mathrm{p}=0.196\end{array}$ \\
\hline Marital status & $\begin{array}{l}\text { Single } \\
\text { Married }\end{array}$ & $\begin{array}{l}564 \\
274\end{array}$ & $\begin{array}{l}55.35 \pm 13.26 \\
55.46 \pm 12.45\end{array}$ & $\begin{array}{l}\mathrm{U}=77072.50 \\
\mathrm{p}=0.953\end{array}$ \\
\hline Education Level & $\begin{array}{l}\text { Primary education } \\
\text { Secondary education } \\
\text { Tertiary education } \\
\text { Master } \\
\text { Doctorate }\end{array}$ & $\begin{array}{l}34 \\
87 \\
499 \\
205 \\
13\end{array}$ & $\begin{array}{l}60.32 \pm 13.66 \\
58.16 \pm 12.55 \\
54.66 \pm 13.18 \\
55.05 \pm 12.14 \\
56.76 \pm 16.35\end{array}$ & $\begin{array}{l}\mathrm{KW}=12.871 \\
\mathrm{p}=0.012\end{array}$ \\
\hline Occupation & $\begin{array}{l}\text { Civil Servant } \\
\text { Health worker } \\
\text { Pensioners } \\
\text { Business owner/trader } \\
\text { Unemployed } \\
\text { Student } \\
\text { Others }\end{array}$ & $\begin{array}{l}119 \\
50 \\
28 \\
82 \\
104 \\
353 \\
102\end{array}$ & $\begin{array}{l}54.13 \pm 12.79 \\
55.30 \pm 10.67 \\
52.21 \pm 14.35 \\
56.70 \pm 11.27 \\
56.76 \pm 12.68 \\
55.35 \pm 13.98 \\
55.42 \pm 11.93\end{array}$ & $\begin{array}{l}\mathrm{KW}=5.877 \\
\mathrm{p}=0.437\end{array}$ \\
\hline Can you receive COVID-19 vaccine? & $\begin{array}{l}\text { Yes } \\
\text { No }\end{array}$ & $\begin{array}{l}473 \\
365\end{array}$ & $\begin{array}{l}56.84 \pm 10.90 \\
53.50 \pm 15.09\end{array}$ & $\begin{array}{l}U=76454.00 \\
p=0.004\end{array}$ \\
\hline Are you against the COVID-19 vaccine? & $\begin{array}{l}\text { Yes } \\
\text { No }\end{array}$ & $\begin{array}{l}101 \\
737\end{array}$ & $\begin{array}{l}51.32 \pm 15.06 \\
55.94 \pm 12.59\end{array}$ & $\begin{array}{l}\mathrm{U}=30767.50 \\
\mathrm{p}=\mathbf{0 . 0 0 5}\end{array}$ \\
\hline $\begin{array}{l}\text { Do you think the COVID-19 vaccine will } \\
\text { be effective? }\end{array}$ & $\begin{array}{l}\text { Yes } \\
\text { No }\end{array}$ & $\begin{array}{l}701 \\
137\end{array}$ & $\begin{array}{l}56.64 \pm 12.33 \\
48.94 \pm 14.36\end{array}$ & $\begin{array}{l}\mathrm{U}=32619.50 \\
\mathrm{p}=\mathbf{0 . 0 0 0}\end{array}$ \\
\hline $\begin{array}{l}\text { Can you receive the COVID vaccine if it } \\
\text { is free? }\end{array}$ & $\begin{array}{l}\text { Yes } \\
\text { No }\end{array}$ & $\begin{array}{l}550 \\
288\end{array}$ & $\begin{array}{l}57.24 \pm 12.62 \\
51.85 \pm 12.97\end{array}$ & $\begin{array}{l}\mathrm{U}=\mathbf{5 7 7 0 0 . 0 0} \\
\mathrm{p}=\mathbf{0 . 0 0 0}\end{array}$ \\
\hline
\end{tabular}

Source: Online field survey 2020.

Table 5. Relationship Between Age, Health Literacy,Vaccine Reliability Scale Total Score Means and Sub-Dimensions

\begin{tabular}{|c|c|c|c|c|c|c|c|c|c|c|c|}
\hline & & (1) & (2) & (3) & (4) & $(5)$ & (6) & (7) & (8) & (9) & (10) \\
\hline $\begin{array}{l}\text { Health Literacy Scale Total Score } \\
\text { Means (1) }\end{array}$ & $\begin{array}{l}\mathbf{r} \\
\mathbf{p}\end{array}$ & - & & & & & & & & & \\
\hline Access to Information (2) & $\begin{array}{l}\mathbf{r} \\
\mathbf{p}\end{array}$ & $\begin{array}{l}.892^{*} \\
.000\end{array}$ & & & & & & & & & \\
\hline Understanding Information (3) & $\begin{array}{l}\mathbf{r} \\
\mathbf{p}\end{array}$ & $\begin{array}{l}.941^{*} \\
.000\end{array}$ & $\begin{array}{l}.798^{*} \\
.000\end{array}$ & & & & & & & & \\
\hline Value Setting (4) & $\begin{array}{l}\mathbf{r} \\
\mathbf{p}\end{array}$ & $\begin{array}{l}.956^{*} \\
.000\end{array}$ & $\begin{array}{l}.797^{*} \\
.000\end{array}$ & $\begin{array}{l}.859^{*} \\
.000\end{array}$ & & & & & & & \\
\hline Application (5) & $\begin{array}{l}\mathbf{r} \\
\mathbf{p}\end{array}$ & $\begin{array}{l}.920^{\star} \\
.000\end{array}$ & $\begin{array}{l}.768^{*} \\
.000\end{array}$ & $\begin{array}{l}.837^{*} \\
.000\end{array}$ & $\begin{array}{l}.858^{*} \\
.000\end{array}$ & & & & & & \\
\hline $\begin{array}{l}\text { Vaccine Reliability Scale Total Score } \\
\text { Means (6) }\end{array}$ & $\begin{array}{l}\mathbf{r} \\
\mathbf{p}\end{array}$ & $\begin{array}{l}-.257^{*} \\
.000\end{array}$ & $\begin{array}{l}-.197^{*} \\
.000\end{array}$ & $\begin{array}{l}-.229^{*} \\
.000\end{array}$ & $\begin{array}{l}-.234^{*} \\
.000\end{array}$ & $\begin{array}{l}-.249^{*} \\
.000\end{array}$ & & & & & \\
\hline Vaccine Benefit Perception (7) & $\begin{array}{l}\mathbf{r} \\
\mathbf{p}\end{array}$ & $\begin{array}{l}-.374^{*} \\
.000\end{array}$ & $\begin{array}{l}-.307^{*} \\
.000\end{array}$ & $\begin{array}{l}-.340^{*} \\
.000\end{array}$ & $\begin{array}{l}-.342 * \\
.000\end{array}$ & $\begin{array}{l}-.361^{*} \\
.000\end{array}$ & $\begin{array}{l}.852^{*} \\
.000\end{array}$ & & & & \\
\hline Vaccine Harm Perception (8) & $\begin{array}{l}\mathbf{r} \\
\mathbf{p}\end{array}$ & $\begin{array}{l}.171^{*} \\
.000\end{array}$ & $\begin{array}{l}.163^{*} \\
.000\end{array}$ & $\begin{array}{l}.155^{*} \\
.000\end{array}$ & $\begin{array}{l}.173^{*} \\
.000\end{array}$ & $\begin{array}{l}.171^{*} \\
.000\end{array}$ & $\begin{array}{l}.527 * \\
.000\end{array}$ & $\begin{array}{l}.141^{*} \\
.000\end{array}$ & & & \\
\hline Trust in Healthcare Professionals (9) & $\begin{array}{l}\mathbf{r} \\
\mathbf{p}\end{array}$ & $\begin{array}{l}-.409^{*} \\
.000\end{array}$ & $\begin{array}{l}-.336^{*} \\
. \mathbf{0 0 0}\end{array}$ & $\begin{array}{l}-.362^{*} \\
.000\end{array}$ & $\begin{array}{l}-.399^{*} \\
.000\end{array}$ & $\begin{array}{l}-.378^{*} \\
.000\end{array}$ & $\begin{array}{l}.747^{*} \\
.000\end{array}$ & $\begin{array}{l}.612^{*} \\
.000\end{array}$ & $\begin{array}{l}.612^{*} \\
.000\end{array}$ & & \\
\hline Age (10) & $\begin{array}{l}\mathbf{r} \\
\mathbf{p}\end{array}$ & $\begin{array}{l}-.039 \\
.258\end{array}$ & $\begin{array}{l}-.055 \\
.112\end{array}$ & $\begin{array}{l}.003 \\
.936\end{array}$ & $\begin{array}{l}-.061 \\
.075\end{array}$ & $\begin{array}{l}-.004 \\
.913\end{array}$ & $\begin{array}{l}-.025 \\
.469\end{array}$ & $\begin{array}{l}.023 \\
.505\end{array}$ & $\begin{array}{l}-.103^{*} \\
.003\end{array}$ & $\begin{array}{l}.039 \\
.259\end{array}$ & \\
\hline
\end{tabular}


conducted is higher than in other countries.

The total mean scores from the Health Literacy Scale were found to be significantly higher among men $(p<0.05)$. There are similar studies in the literature. ${ }^{17,18}$

The total mean scores from the Health Literacy Scale were found to be significantly higher among civil servant $(p<0.05)$. In the literature, health literacy was found to be higher among those working in an income generating job.19,20 In addition, it has been determined that the health personnel have a moderate level of health literacy. This situation suggests that it may be due to the fact that health personnel focus on their work in the unit they are located in and have little interest in other areas.

The total mean scores from the Health Literacy Scale were found to be significantly higher among those who do not think the COVID-19 vaccine will be effective $(p<0.05)$. Individuals may think so, since information about the vaccine has not been obtained from the right sources and concrete steps have not yet been taken for the coronavirus vaccine.

The total mean scores from the Health Literacy Scale were found to be significantly higher among those who will not receive the COVID vaccine if it is free $(p<0.05)$. Health literacy can also be affected by the economic situation, ${ }^{19,20}$ it is thought that individuals have a negative attitude regardless of their wages.

The total mean scores from the Vaccine Reliability Scale were found to be significantly higher among the Indians $(\mathrm{p}<0.05)$. This situation suggests that awareness of individuals' attitude towards vaccines has increased due to the fact that India is a very crowded country and the epidemic rate is higher.

The total mean scores from the Vaccine Reliability Scale were found to be significantly higher among those who receive COVID-19 vaccine $(p<0.05)$. It is thought that as the attitude towards the vaccine increases, they also take a more positive approach to the coronavirus vaccine.

The total mean scores from the Vaccine Reliability Scale were found to be significantly higher among those who do not against the COVID-19 vaccine $(p<0.05)$. As the attitude towards vaccination increases, anti-vaccination decreases and it is thought that the attitude towards vaccination increases positively.

The total mean scores from the Vaccine Reliability Scale were found to be significantly higher among those who think the COVID-19 vaccine will be effective $(p<0.05)$. Individuals who have a positive attitude towards the vaccine can be interpreted as they think the vaccine will be effective.

The total mean scores from the Vaccine Reliability Scale were found to be significantly higher among those who will receive the COVID vaccine if it is free $(p<0.05)$. Since the attitude towards the vaccine is positive, it suggests that if the vaccine is produced and free of charge, they will be in high demand.

It was found that there was a statistically significant negative correlation between Health Literacy scale total score mean and Vaccine Reliability Scale Total Score Means and two Vaccine Reliability Scale sub dimensions; Vaccine Benefit Perception and Trust in Healthcare Professionals $(p<0.05)$. When welook at the history of vaccines, vaccines have been blamed many times in the period that has passed until today. However, most of the charges are mythologically based. The accusations made with more "medical looking" explanations could not be proven clinically. For example, studies have been carried out on the claim that the mercury in the vaccines causes autism. However, no study has proven this claim. Nevertheless, the US government announced that it removed mercury from vaccines in 2001, fearing that the vaccination rates will decrease. ${ }^{21}$ However, parents believe that there is still mercury in vaccines and that it is associated with autism in the long term. Similarly, he believes that aluminum and other chemicals accumulate in the body and will lead to chronic diseases in the future. There are claims stating that aluminum is a neurotoxin and that vaccines containing aluminum in infants whose brain development is not yet completed may cause neurodevelopmental and autoimmune diseases. ${ }^{22}$ In laboratory studies, the toxic effects of aluminum and mercury on neuron cell cultures were mentioned, and another study claimed that some sheep developed postvaccination encephalitis. ${ }^{23,24}$ However, clinically, there are many cases of encephalitis and meningitis prevented by vaccines, as there are no case reports of vaccine-induced encephalitis. 
Studies have shown that in the last 20 years, the most measles cases in the United States were seen in 2014, and most of these cases were as a result of individual vaccine rejection. ${ }^{25}$ Similarly, there was a significant increase in measles case reports in 2011 in Europe, and it was determined that $85 \%$ of them were unvaccinated or unregistered..$^{26}$ Another concern is that vaccines have side effects. In the study of parents who refused vaccines in Venezuela, the side effects of vaccines and the idea that it is unnecessary to give more than one vaccine came to the fore. ${ }^{27}$ Yes, vaccines and other substances added to increase the effectiveness of vaccines and prevent their deterioration may have many side effects, from simple pain to anaphylaxis. However, the side effects of vaccines are much less frequent and milder compared to the disease itself. ${ }^{28}$ In addition, knowing that all medical interventions carry risks and informing patients scientifically by healthcare personnel can reduce concerns about this issue. Considering the benefit-harm balance, studies are in favor of vaccination. ${ }^{29}$ In order to increase the safety and efficacy of vaccines, studies to reduce their side effects may be needed, but considering the benefits, it is beyond the mind and science that this situation leads to vaccine rejection.

Studies have shown that religious beliefs and religious leaders in Africa, Afghanistan and India are effective in vaccination. Jarett et al. According to the WHO 'Vaccine Hesitations Working Group' analysis in 2015, the fact that people describe themselves as more knowledgeable and increased awareness is at the forefront of the trust questions about vaccines, and if we do not count the availability of the vaccine, the guidance of religious and philosophically active people is seen as a problem that requires mutual dialogue that is difficult to solve. ${ }^{30}$ It is thought that the negative attitude of the group with high health literacy towards vaccination is due to the side effects of the individuals, possible complications and religious philosophical knowledge.

\section{Conclusions}

The Health Literacy total score mean was found to be statistically significantly higher among Nepal nationality, male, civil servant and those who do not think the COVID-19 vaccine will be effective and those who do not think they will receive the COVID vaccine if it is free $(p<0.05)$. The vaccine reliability scale total score mean was found to be statistically significantly higher among India nationality, those who receive COVID-19 vaccine, who do not against the COVID-19 vaccine, who think the COVID-19 vaccine will be effective and who will receive the COVID-19 vaccine if it is free $(p<0.05)$. It is recommended to conduct the study in a larger groups.

\section{Conflict of interest}

The authors declared that there are no potential conflicts of interest with respect to the research, authorship, and/or publication of this article.

\section{Contribution of Authors}

Concept-G. O., M.S.Y., M.Y.; Design-M.A.A., Y.S.; Supervision - S.M., A.T., M.T.; Materials A.U., R.B.K., K.G., A.G.; Data collection \&/or processing-M.S.Y., M.Y., S.M., A.T., M.T., A.U., R.B.K., K.G., A.G., N.A.R., A.R. ; Analysis and/ or interpretation - U.M., M.S.Y., M.Y.; Literature search - D.G., M.Y., M.S.Y. Writing - M.S.Y., M.Y.; Critical review -G.O.

\section{References}

1. Riedel S, editor Edward Jenner and the history of smallpox and vaccination. Baylor University Medical Center Proceedings; 2005: Taylor \& Francis. https://doi. org/10.1080/08998280.2005.11928028

2. Ullmann A. Pasteur-Koch: Distinctive ways of thinking about infectious diseases. Microbe (Washington, D.C.) 2007;2(8):383-7.

3. World Health Organization. Immunization, Vaccines and Biologicals. Available at: https://www.who.int/ immunization/global_vaccine_action_plan/GVAP_ doc_2011_2020/en/. Accessed Nov, 2020.

4. Andre FE, Booy R, Bock HL, Clemens J, Datta SK, John $\mathrm{TJ}$, et al. Vaccination greatly reduces disease, disability, death and inequity worldwide. Bulletin of the World health organization. 2008;86:140-6. doi: 10.2471/blt.07.040089.

5. Johnson DR, Nichol KL, Lipczynski K. Barriers to adult immunization. Am J Med. 2008 Jul;121(7 Suppl 2):S2835. doi: 10.1016/j.amjmed.2008.05.005.

6. Toprak D, Akan H, Köksal İ, Sargın M. Erişkin aşılaması, uygulamadaki sorunlar ve çözüm önerileri, aile hekimlerinin erişkin aşılamasındaki rolü. Türkiye Aile Hekimliği Dergisi. 2018;22:166-74.

7. Salmon DA, Moulton LH, Omer SB, DeHart MP, Stokley S, Halsey NA. Factors associated with refusal of childhood vaccines among parents of school-aged children: a case-control study. Arch Pediatr Adolesc Med. 2005 May;159(5):470-6. doi: 10.1001/archpedi.159.5.470. 
8. Ropeik D. How society should respond to the risk of vaccine rejection. Hum Vaccin Immunother. 2013 Aug;9(8):1815-8. doi: 10.4161/hv.25250.

9. Sadaf A, Richards JL, Glanz J, Salmon DA, Omer SB. A systematic review of interventions for reducing parental vaccine refusal and vaccine hesitancy. Vaccine. 2013;31:4293-304. doi: 10.1016/j.vaccine.2013.07.013.

10. Patel H, Jeve YB, Sherman SM, Moss EL. Knowledge of human papillomavirus and the hu-man papillomavirus vaccine in European adolescents: a systematic review. Sex Transm Infect. 2016;92:474-9 doi: 10.1136/ sextrans-2015-052341.

11. Lorini C, Santomauro F, Donzellini M, Capecchi L, Bechini A, Boccalini S, Bonanni P, Bonaccorsi G. Health literacy and vaccination: A systematic review. Hum Vaccin Immunother. 2018 Feb 1;14(2):478-488. doi: 10.1080/21645515.2017.1392423.

12. Wilson FL, Baker LM, Nordstrom CK, Legwand C. Using the teach-back and Orem's Self-care Deficit Nursing theory to increase childhood immunization communication among low-income mothers. Issues Compr Pediatr Nurs. Jan-Mar 2008;31(1):7-22. doi: 10.1080/01460860701877142.

13. Castro-Sánchez E, Chang PW, Vila-Candel R, Escobedo AA, Holmes AH. Health literacy and infectious diseases: why does it matter? Int J Infect Dis. 2016 Feb;43:103-110. doi: 10.1016/j.ijid.2015.12.019.

14. Berkman ND, Sheridan SL, Donahue KE, Halpern DJ, Viera A, Crotty K, Holland A, Brasure M, Lohr KN, Harden E, Tant E, Wallace I, Viswanathan M. Health literacy interventions and outcomes: an updated systematic review. Evid Rep Technol Assess (Full Rep). 2011 Mar;(199):1-941.

15. Gilkey MB, Magnus BE, Reiter PL, McRee AL, Dempsey AF, Brewer NT. The Vaccination Confidence Scale: a brief measure of parents' vaccination beliefs. Vaccine. 2014 Oct 29;32(47):6259-65. doi: 10.1016/j.vaccine.2014.09.007.

16. Sørensen K, Van den Broucke S, Pelikan JM, Fullam J, Doyle G, Slonska Z, Kondilis B, Stoffels V, Osborne $\mathrm{RH}$, Brand H; HLS-EU Consortium. Measuring health literacy in populations: illuminating the design and development process of the European Health Literacy Survey Questionnaire (HLS-EU-Q). BMC Public Health. 2013 Oct 10;13:948. doi: 10.1186/1471-2458-13-948.

17. Tanrı̈ver MD, Yıldırım HH, Ready FN, Çakır B, Akalın HE. Sağlık okuryazarlığı araştırması. Ankara 2014. Avaliable at: http://www.sagliksen.org.tr/cdn/uploads/ gallery/pdf/8dcec50aa18c21cdaf86a2b33001a409.pdf. Accessed December 20, 2020.
18. Efteli E, Khorshtd L. İki Farklı Bölüm Öğrencilerinin Sağlık Algılarının Karşılaştırılması İki Farklı Bölüm Öğrencilerinin Sağlık Algılarının Karşılaştırılması. Ege Üniversitesi Hemşirelik Fakültesi Dergisi, 2016; 32(2): 1-10.

19. Rivero-Méndez M, Suárez-Pérez EL, Solís-Báez SS. Measuring health literacy among people living with HIV who attend a community-based ambulatory clinic in Puerto Rico. P R Health Sci J. 2015 Mar;34(1):31-7.

20. Zhang Y, Zhang F, Hu P, Huang W, Lu L, Bai R, Sharma M, Zhao Y. Exploring Health Literacy in Medical University Students of Chongqing, China: A CrossSectional Study. PLoS One. 2016 Apr 6;11(4):e0152547. doi: 10.1371/journal.pone.0152547.

21. Offit PA. Thimerosal and vaccines-a cautionary tale. $\mathrm{N}$ Eng1 J Med. 2007 Sep 27;357(13):1278-9. doi: 10.1056/ NEJMp078187.

22. Miller NZ. Aluminium in Childhood Vaccines is Unsafe. J Am Physic Surg 2016;21(4):109-117.

23. Haley BE. Mercury toxicity: genetic susceptibility and synergistic effects. Medical Veritas 2005;2(2):535-542. Available at: http://www.1796kotok. com/pdfs/haley.pdf. Accessed Nov, 2020. doi: 10.1588/medver.2005.02.00067

24. Luján L, Pérez M, Salazar E, Álvarez N, Gimeno M, Pinczowski P, Irusta S, Santamaría J, Insausti N, Cortés Y, Figueras L, Cuartielles I, Vila M, Fantova E, Chapullé JL. Autoimmune/autoinflammatory syndrome induced by adjuvants (ASIA syndrome) in commercial sheep. Immunol Res. 2013 Jul;56(2-3):317-24 . doi: 10.1007/ s12026-013-8404-0.

25. Centers for Disease Control and Prevention. Measles cases in the United States reach 20-year high. Available at: https://www. cdc.gov/media/releases/2014/ p0529measles.html. Accessed Nov, 2020.

26. Cottrell S, Roberts RJ. Measles outbreak in Europe. BMJ. 2011 Jun 15;342:d3724. doi: 10.1136/bmj.d3724.

27. Burghouts J, Del Nogal B, Uriepero A, Hermans PW, de Waard JH, Verhagen LM. Childhood Vaccine Acceptance and Refusal among Warao Amerindian Caregivers in Venezuela; A Qualitative Approach. PLoS One. 2017 Jan 20;12(1):e0170227. doi: 10.1371/journal.pone.0170227

28. Özen M. Doğan N. Aşı-Hastalık ilişkisi: Söylenti mi, Gerçek mi?. Klinik Gelişim 2012;25, 16-20.

29. Argüt N., Yetim A., Gökçay G. Aş1 Kabülünü Etkileyen Faktörler. Çocuk Derg 2016;16(1-2), 16-24.

30. Jarrett C, Wilson R, O'Leary M, Eckersberger E, Larson HJ; SAGE Working Group on Vaccine Hesitancy. Strategies for addressing vaccine hesitancy - A systematic review. Vaccine. 2015 Aug 14;33(34):4180-90. doi: 10.1016/j.vaccine.2015.04.040. 